\title{
Custos e riscos financeiros da implementação das terapias gênicas e celulares em doenças raras no setor público
}

\begin{abstract}
Alberto Guilherme Faria de OLIVEIRA: Faculdade de Saúde Pública - FSP, Universidade de São Paulo - USP. ORCID: 0000-0001-5759-8697

Jaqueline Vilella BULGARELI: Faculdade de Saúde Pública - FSP, Universidade de São Paulo USP. ORCID: 0000-0001-7810-0595
\end{abstract}

\section{Resumo}

Com o avanço da medicina, novas tecnologias vêm sendo desenvolvidas pela Indústria Farmacêutica com o objetivo de proporcionar a erradicação de doenças e/ou o aumento significativo da qualidade de vida dos pacientes. Atualmente, os produtos de terapia avançada (PTA) representados pelas categorias terapia celular avançada, engenharia tecidual e terapia gênica têm demonstrado grande potencial terapêutico, através de resultados de estudos clínicos. Apesar da maioria dos estudos em andamento terem como foco a oncologia, recentes lançamentos da indústria tiveram como alvo as doenças raras. Além destas áreas terapêuticas, acredita-se que estas terapias poderão ser aplicadas em outras áreas como doenças crônicas que atingem o Sistema Nervoso Central (SNC), como a doença de Alzheimer e de Parkinson e outras, como a hemofilia. Apesar de alguns países estarem realizando pesquisas e investimentos para o desenvolvimento dos PTAs, somente uma pequena parcela destas encontram-se disponíveis como alternativa terapêutica. Ainda que existam expectativas que tais tecnologias sejam capazes de eliminar enfermidades consideradas incuráveis, muito se discute quanto aos desafios em jogo para viabilizarem a sua implementação nos sistemas de saúde. Além dos desafios para a execução dos estudos clínicos, padronização do modelo de produção e da regulação, existem incertezas quanto a sua eficácia e segurança no longo prazo, sendo necessário o desenvolvimento de plataformas para o monitoramento dos pacientes, a fim de se obterem dados que justifiquem a sua utilização, bem como estudos de custo-efetividade e determinação de medidas de eficácia. Uma vez que os valores individuais das PTAs são elevados e, em contrapartida, o orçamento em saúde dos países é limitado, estudos de mundo real serão fundamentais para se discutir sobre uma estratégia de financiamento com o foco em uma estrutura de valor compartilhado. O objetivo deste trabalho foi investigar na literatura estudos relacionados aos custos e riscos financeiros aos governos com a implementação das terapias gênicas e celulares em doenças raras no setor público. Este método foi realizado à partir de uma revisão integrativa e sistematizada da literatura com o propósito de direcionar o pesquisador a prática, se respaldando de embasamento científico. Em Esta metodologia consiste em sintetizar as pesquisas disponíveis em bancos de dados sobre o tema escolhido, analisar criticamente os resultados de busca e decidir pela inclusão ou exclusão dos artigos científicos relacionados. A revisão foi iniciada a partir da pergunta de pesquisa, definida como "O que a literatura científica apresenta sobre os desafios de implementação das terapias gênicas e celulares no setor público, com foco nas doenças raras, bem como os seus custos e riscos financeiros aos governos?". A pergunta serviu como base para a definição dos descritores de interesse nas plataformas http://decs.bvs.br/ Descritores em ciências da saúde, e da plataforma Mesh https://meshb.nlm.nih.gov/ Medical Subject Headings. A busca e seleção dos artigos científicos utilizou os descritores 
relacionados ao tema de pesquisa. Dos resultados obtidos, os descritores foram combinados em polos a fim de se construir a sintaxe da pesquisa de tal maneira, que fosse possível obter os resultados de forma abrangente, sistematizada e reprodutível. Os polos foram divididos em fenômeno, população e contexto, dialogando com a pergunta de pesquisa. Dentro de cada polo, adotou-se os operadores booleanos "OR" com o objetivo de expandir a busca dos artigos. Por outro lado, com a utilização do operador booleano "AND", os resultados foram restritos e mapeados estrategicamente para a obtenção dos resultados de interesse, relacionados ao tema do estudo. A busca bibliográfica foi realizada no portal Biblioteca Virtual em Saúde (BVS) - http://bvsalud.org/, Pubmed https://pubmed.ncbi.nlm.nih.gov/ e através do Portal de Periódicos da CAPES, foram acessados as bases de dados Scopus - https://www.scopus.com/ e Embase - https://wwwembase.ez67.periodicos.capes.gov.br/. Através da aplicação do operador booleano "AND , foi obtida a sintaxe final composta pela conexão dos pólos fenômeno, contexto e população: mh:((mh:(mh:("Terapia Genetica" OR "Imunoterapia Adotiva"))) AND (mh:(mh:("Doencas raras" OR "humanos" OR "medicina estatal" OR "assistencia de saude universal" $O R$ "programas nacionais de saude" $O R$ "politica de saude" $O R$ "sistema único de saude" $O R$ "cobertura do seguro de saude" OR "programas governamentais" OR "Acesso universal aos servicos de saude" $O R$ "Estrategias para Cobertura Universal de saude" $O R$ "Ensaios clínicos como Assunto" OR "Parcerias Publico-privadas"))) AND (mh:(mh:("Custos e analise de Custo" OR "Analise custo-beneficio" OR "Custos de medicamentos" OR "Custos de Cuidados de Saude" OR "Participacao no Risco Financeiro" OR "Avaliacao de Resultados em cuidados de saude" OR "Mecanismo de reembolso"))). Através do método de busca adotado, obteve-se 77 artigos na BVS, 1374 na Pubmed, 194 no Scopus e 1110 no Embase. Foram extraídos artigos que abordavam os aspectos financeiros e científicos das terapias gênicas e celulares, no âmbito dos sistemas de saúde, resultados de estudos clínicos, eventos adversos, bem como aspectos de produção e regulação destes produtos. Os critérios de exclusão consistiam em publicações além dos últimos 10 anos, os idiomas nas línguas inglesa, portuguesa e espanhola. Para evitar a duplicidade de artigos, foi utilizado o programa Zotero. Ao final, foram selecionados até o momento 34 artigos para leitura na íntegra e destes 34, foram adicionados 6 artigos da literatura cinzenta como complemento ao conteúdo do trabalho. Os demais resultados encontrados foram excluídos por não apresentarem temática direta com a pesquisa proposta. Como considerações finais, notase o interesse da indústria farmacêutica na medicina personalizada, através do lançamento dos produtos de terapia avançada (PTA). Estas terapias se mostraram eficientes em doenças raras e apesar dos últimos lançamentos terem sido para esta população, a maioria dos estudos clínicos em andamento têm como foco a oncologia e deverão ser comercializados em breve. Não obstante, foram observados diversos incentivos governamentais para o seu desenvolvimento. Todavia, devido ao seu valor individual, muito têm se discutido entre agências de ATS e os fabricantes, possíveis modelos de financiamento para promoção do acesso, e o modelo que mais têm se destacado é o de compartilhamento de risco, adotado em alguns países da Europa. Além do aspecto financeiro, também se observou a importância da harmonização entre as agências regulatórias para a aprovação destas terapias, bem como a padronização dos estudos clínicos e métodos de produção.

Descritores: Terapia Genética; Alocação de Custos; Doenças Raras; Risco Financeiro.

Keywords: Genetic Therapy; Cost Allocation; Rare Diseases; Financial Risk.

Palabras-claves: Terapia Genética; Asignación de Costos; Enfermedades Raras; Riesgo Financiero. 